$\mathrm{t}^{1} / 2$ de $\beta, \mathrm{t}^{1} / 2$ de $\gamma, \mathrm{AUC}^{0-\infty}, \mathrm{Cl} / \mathrm{f} / \mathrm{kg}$ e Vd/f/kg. Com base nas concentrações maternas e fetais no momento do nascimento, foi determinada a relação feto/materna desse fármaco.

Resultados: os parâmetros farmacocinéticos encontrados foram: $\mathrm{t}^{1} / 2$ de $\alpha$ de $13,5 \mathrm{~min}, \mathrm{t}^{1} / 2$ de $\beta$ de $192,5 \mathrm{~min}$, $\mathrm{t}^{1} /{ }_{2}$ de $\gamma$ de $620 \mathrm{~min}, \mathrm{AUC}^{0-\infty}$ de 137,404 ng.min $/ \mathrm{mL}, \mathrm{Cl} /$ f de $464,984 \mathrm{~mL} / \mathrm{min}, \mathrm{Vd} / \mathrm{f}$ de $299,974 \mathrm{~L}, \mathrm{Cl} / \mathrm{f} / \mathrm{kg}$ de $6,875 \mathrm{~mL} / \mathrm{min} / \mathrm{kg}$ e $\mathrm{Vd} / \mathrm{f}, / \mathrm{kg}$ de $4,441 \mathrm{~L} / \mathrm{kg}$. O tempo de latência entre a administração da droga e o nascimento foi de 28,5 min, sendo que, no momento do parto, a concentração plasmática materna foi de $0,310 \mathrm{ng} /$
$\mathrm{mL}$ e a fetal de $0,245 \mathrm{ng} / \mathrm{mL}$, com relação feto/materna mediana de 0,892 .

Conclusões: a fentanila apresenta rápida passagem do espaço epidural para a corrente sangüínea e curto período de tempo para equilíbrio entre esses setores. O estudo evidenciou transferência placentária da fentanila em razões de aproximadamente 90\% para fentanila, alertando para a elevada transferência deste fármaco através da barreira placentária.

PALAVRAS-CHAVE: Anestesia obstétrica. Cesárea via epidural. Farmacocinética. Transferência placentária.

\title{
Mortalidade Materna na Cidade de São Paulo de 1995 A 1999, com Ênfase em Hipertensão Arterial
}

\author{
Maternal Mortality in the City of Sao Paulo, from 1995 to 1999, with Emphasis on Hypertension
}

Autor: Carlos Eduardo Pereira Vega

Orientador: Prof. Dr. Soubhi Kahhale

Tese de Doutorado apresentada ao Departamento de Obstetrícia e Ginecologia da Faculdade de Medicina da Universidade de São Paulo, em 26 de maio de 2004.

Analisou-se a mortalidade materna na cidade de São Paulo de 1995 a 1999, com ênfase nas mortes decorrentes de complicações da hipertensão arterial. Foi realizada uma comparação entre a casuística oficial de morte materna com a que foi apurada pelo Comitê de Mortalidade Materna Municipal, objetivando avaliar os resultados obtidos em ambos os métodos de coleta de dados. O método empregado pelo Comitê se mostrou de maior valor para a realização de estudos específicos sobre as causas determinantes do óbito materno e dentre os 800 casos identificados no período avaliado, encontramos 142 mortes maternas ocasionadas por distúrbios hipertensivos. Os casos foram estudados segundo a faixa etária, cor, local de residência e óbito, subnotificação do óbito materno, responsabilidade do atendimento (público ou privado), atendimento hospitalar, intervenções obstétricas durante a internação e discutida a evitação da morte materna por hipertensão arterial. Para avaliação da qualidade e estrutura do atendimento hospitalar foi elaborado um indicador denominado Índice de Letalidade Hospitalar (ILH). O setor público foi responsável por $80,6 \%$ dos óbitos decorrentes de complicações hipertensivas no município de São Paulo. Verificaram-se falhas no atendimento, tais como: planejamento familiar insatisfatório, atendimento pré-natal inadequado e ineficiente, presença de grandes deslocamentos à procura de vaga hospitalar, existência de hospitais com estrutura desprovida de condições ao atendimento da gestante hipertensa e suas complicações, acompanhamento pós-natal deficitário e subutilização do sulfato de magnésio na prevenção e tratamento das crises convulsivas. São sugeridas medidas para o atendimento apropriado da gestante e puérpera hipertensa, visando à redução da morbimortalidade materna.

PALAVRAS-CHAVE: Mortalidade materna. Hipertensão arterial. Eclâmpsia. Complicações da gravidez.

\section{Imunofenótipo Leucocitário no Câncer de Mama e sua Associação com Indicadores Proǵnósticos}

\section{The Leukocytic Immunophenotype}

Autor: Alexandre Henrique Macchetti

Orientador: Prof. Dr. Heitor Ricardo Cosiski Marana

Dissertação de Mestrado apresentada ao Departamento de Ginecologia e Obstetrícia da Faculdade de Medicina de Ribeirão Preto da Universidade de São Paulo, em 18 de maio de 2004.

Objetivos: analisar o infiltrado leucocitário do câncer de mama utilizando a imunofenotipagem, e correlacionar esta composição com o tamanho e grau histológico tumoral e acometimento linfonodal axilar por metástase. 
Métodos: 35 pacientes do Ambulatório de Mastologia do HC-FMRP-USP com carcinoma mamário tiveram amostra tumoral submetida à digestão por solução enzimática para extração dos leucócitos, e a seguir o imunofenótipo dos leucócitos analisado por citometria de fluxo com dupla marcação $\left(\mathrm{CD}_{3}, \mathrm{CD}_{4}, \mathrm{CD}_{8}, \mathrm{CD}_{19}, \mathrm{CD}_{14}\right.$ e $\mathrm{CD}_{16 / 56}$ ). Os resultados foram correlacionados com indicadores prognósticos clínico-patológicos.

Resultados: houve predominância dos linfócitos T e macrófagos (médias de 19,9 e 13,3\%) com baixos percentuais de linfócitos B e células Natural Killer (médias 3,45 e 4,6\%) no infiltrado. A infiltração por linfócitos $\mathrm{T}$ esteve diretamente correlacionada com o tamanho tumoral e envolvimento nodal em tumores menores que $5 \mathrm{~cm}$. O percentual de linfócitos $\mathrm{T}$ foi maior do que o de macrófagos em tumores menores que $5 \mathrm{~cm}$ e com acometimento axilar. O número de linfonodos axilares com metástase foi correlacionado com o infiltrado linfocitário T. Não houve correlação entre o grau histológico tumoral e os subtipos de leucócitos intratumorais, nem correlação da razão CD4/ CD8>1 com acometimento nodal.

Conclusões: a associação do infiltrado linfocitário T maior nos tumores em tumores menores que $5 \mathrm{~cm}$, e dentro destes tumores, associação com metástase axilar, sugere que os linfócitos $\mathrm{T}$ possam ser manipulados no microambiente tumoral favorecendo a disseminação das células tumorais.

PALAVRAS-CHAVE: Mama: carcinoma. Linfócitos infiltrantes de tumor. Macrófagos. Citometria de fluxo.

Hiperestimulação Ovariana Controlada com FSH Exclusivo Seguido de Estimulaçãocom HCG ouhMG

\section{Controlled Ovarian Stimulation with FSH Alone Followed by Stimulation With hCG or hMG}

Autora: Mariana Kefalás Oliveira Gomes

Orientador: Prof. Dr. Rui Alberto Ferriani

Dissertação de Mestrado apresentada à Faculdade de Medicina de Ribeirão Preto - Universidade de São Paulo Departamento de Ginecologia e Obstetrícia, em 6 de julho de 2004.

Objetivos: o objetivo desse estudo prospectivo e controlado é avaliar se o LH sob forma de baixa dose de gonadotrofina coriônica humana (hCG) é similar à gonadotrofina menopausal humana (hMG) na fase folicular tardia de pacientes em hiperestimulação ovariana controlada (HOC).

Casuística e Métodos: trinta e quatro mulheres normovulatórias com indicação de ICSI foram randomicamente direcionadas a dois diferentes protocolos de HOC (17 em cada). Todas as pacientes foram suprimidas com análogos do GnRH e receberam FSH recombinante $(200 \mathrm{IU} / \mathrm{d})$ até que se obtivessem folículos entre 13-14 mm de diâmetro médio. A partir de então, constituíram-se dois grupos: HOC com hCG $(200 \mathrm{IU} / \mathrm{d})$ (grupo hCG) e HOC com hMG (225IU/d) (grupo hMG) até que os parâmetros para administração do hCG préovulatório fossem atingidos. A monitorização foi realizada através de ultra-sonografia transvaginal e dosagem sérica de estradiol, progesterona e testosterona. Resultados: o número de folículos menores que 10, 10 a 14 e maiores que $14 \mathrm{~mm}$ e tempo de HOC (em dias) foram similares em ambos os grupos. De 17 pacientes hiperestimuladas com hCG, 14 apresentaram progesterona sérica $>1,5 \mathrm{ng} / \mathrm{mL}$ no dia do hCG pré-ovulatório, comparado com sete pacientes no grupo hMG. As taxas de gravidez clínica foram semelhantes nos grupos hCG e hMG (52.9\% e 33.3\%, respectivamente). O custo total do tratamento por paciente no grupo hCG foi significativamente inferior que no grupo hMG (R\$ $2363,00 \pm 409,70$ vs $\mathrm{R} \$ 1949,00 \pm 292,40$, respectivamente; $p$ 0,0019).

Conclusões: LH na forma de baixa dose de hCG na fase folicular tardia apresentou o mesmo padrão de desenvolvimento folicular que o hMG. O protocolo utilizando hCG produziu taxa de gestação similar àquela evidenciada pela HOC com hMG na fase folicular tardia, mesmo apresentando níveis séricos elevados de progesterona no dia do hCG pré-ovulatório.

PALAVRAS-CHAVE: Hormônio luteinizante. Gonadotrofina coriônica humana. hMG. Hiperestimulação ovariana controlada. 